\title{
REVOLUTION UND LEGALITÄT
}

\author{
EIN UNVEROFFENTLICHTER BRIEF \\ FRIEDRICH ENGELS' \\ AN RICHARD FISCHER
}

Im Januar 1895 war der Geschäftsführer des Verlags „Buchhandlung Vorwärts”, Richard Fischer, „beim Durchblättern alter Zeitungen”1 auf Marxens Artikel „Die Juniniederlage 1848”, „Der 13. Juni 1849” und „Folgen des 13. Juni 1849" gestoßen, welche 1850 in den ersten drei Heften der Neuen Rheinischen Zeitung. Politisch-ökonomische Revue erschienen waren. Am 30. des Monats bat er Engels in einem Brief um dessen Zustimmung zur Herausgabe der völlig unbekannten Marxschen Arbeit in Form einer Broschüre. ${ }^{2}$ Gleichzeitig ersuchte er ihn, eine Einleitung dazu zu verfassen. Da Fischer die Schrift spätestens im März herausbringen wollte - er hielt Engels vor Augen, daß nach einer möglichen Annahme der Umsturzvorlage das Erscheinen der Marxschen Arbeit in Frage gestellt sei -, drängte er auf eine umgehende definitive Antwort. Engels monierte zwar am 2. Februar die Art und Weise, in der er zeitlich unter Druck gesetzt würde, stimmte aber dem Plan Fischers zu und versprach, die gewünschte Einleitung in Angriff zu nehmen. Darüber hinaus erweiterte er die Marxschen Artikel um jene Abschnitte aus der „Revue” des fünften und sechsten Heftes der Neuen Rheinischen Zeitung, die die Ereignisse in Frankreich behandelten. Aus drei Vorschlägen, die Engels machte, wählte Fischer den Titel „Die Klassenkämpfe in Frankreich 1848 bis 1850 ".

$\mathrm{Daß}$ dieser Titel in der wissenschaftlichen und politischen Auseinandersetzung um den Marxismus und die Geschichte der deutschen Sozialdemokratie in den Jahrzehnten seit 1895 einer der meistgenannten aus dem umfangreichen Werk von Karl Marx ist, liegt ausschließlich in der Engelsschen Einleitung begründet. Dabei ging und geht es einmal um die Interpretation dessen, was Engels dort ausgesagt hat, zum anderen um die Bewertung der Tatsache, daß Engels' Text „einiges gelitten unter umsturzvorlagenfurchtsamlichen Bedenken

1 Richard Fischer an Engels, 9.2.1895, IISG, Marx-Engels-Nachlaß, L 1848.

2 Ebenda, L 1852. 
unserer Berliner Freunde, denen ich unter den Umständen wohl Rechnung tragen mußte", wie es in seinem Brief an Karl Kautsky vom 23.3.1895 heißt. ${ }^{1}$

Der Vorwurf, der Engelssche Text sei gegen den Willen des Verfassers verstümmelt und verfälscht worden, ist seit der Veröffentlichung der vom Vorstand gewünschten Streichungen durch Rjazanow ${ }^{2}$ immer wieder erhoben worden. Schon lange vorher, im Jahre 1908, hat Adolf Braun in einem Schreiben an Kautsky geäußert: „Die ,sehr gründlichen' Änderungen der Vorrede von Engels zu den Klassenkämpfen von Marx sind von Richard Fischer gemacht worden. Ich erinnere mich noch sehr genau an die Bürstenabzüge, in denen nur sehr wenig von dem gerettet blieb, was Engels geschrieben hatte." Ein Vergleich der Setzereifahnen mit dem Manuskript zeigt die Unhaltbarkeit dieser Äußerung und macht deutlich, von welch zweifelhaftem Wert für die Geschichtswissenschaft die „sehr genauen” Erinnerungen von Augenzeugen sind. ${ }^{4}$

Neben den schweren an die Adresse des Parteivorstandes gerichteten Vorwürfen stehen die Versicherungen, daß die Streichungen durchaus im Einverständnis mit Engels vorgenommen worden seien. Zur Stützung dieser These wird in erster Linie der Brief Fischers an Engels vom 14. März 1895 herangezogen. ${ }^{5}$ Er ist im Oktober 1926 zusammen mit dem in diesem Zusammenhang bedeutungsvollen Brief an Engels vom 6. März 1895 von Eduard Bernstein in den Sozialistischen Monatsheften veröffentlicht worden. ${ }^{\circ}$ Allerdings zeigt ein

1 Vgl. Friedrich Engels' Briefwechsel mit Karl Kautsky, hrsg. und bearb. von Benedikt Kautsky, Wien 1955, S. 426.

${ }^{2}$ N. Rjazanow, Engels' Einleitung zu Marx' „Die Klassenkämpfe in Frankreich 1848-1850", in: Unter dem Banner des Marxismus, I, 1925, S. 160-165.

${ }^{3}$ Adolf Braun an Karl Kautsky, 5.10.1908, IISG, Kautsky-Nachlaß, D VI, 292.

4 Im übrigen ist es nicht möglich, daß sich Braun hier auf die entstellte Wiedergabe der Engelsschen Gedanken im Vorwärts vom 30. März 1895 bezogen hat (vgl. Engels an Kautsky, 1.4.1895, in: Fr. Engels' Briefwechsel mit Karl Kautsky, a.a.O., S. 429f., und Engels an Paul Lafargue, 3.4.1895, in Marx-Engels, Ausgewählte Briefe, Berlin (O) 1953, S. 586f.), da Fischer nichts damit zu tun hatte, sondern sich im Gegenteil scharf vom Vorgehen Liebknechts distanzierte. Vgl. den Brief an Engels vom 6.4.1895, wo es u.a. heißt: „Was nun die ,Besprechung' im Vorwärts anbetrifft, so reden wir ja darüber noch. Du wunderst Dich eben noch über den „V." - das habe ich mir längst abgewöhnt - ich wunderte mich nur dann, wenn er einmal auf der Höhe seiner Aufgabe stände. Das ist aber nicht zu fürchten, solange die Chefredaktion dauert." (IISG, Marx-EngelsNachlaB, L 1857.)

5 Vgl. z.B. Christian Gneuss, Um den Einklang von Theorie und Praxis, in: Marxismusstudien, zweite Folge, Tübingen 1957, S. 202.

- Eduard Bernstein, Richard Fischer zum Gedächtnis, in : Sozialistische Monatshefte, XXXII. Jahrg., Bd. 63, 1926, S. $676 f$. 
Vergleich der dort unvollständig abgedruckten Briefe mit den Originalen, daß Bernstein nicht nur Unwesentliches ausgelassen hat, sondern auch Passagen, deren Veröffentlichung er offensichtlich scheute. Deshalb werden im Rahmen dieses Aufsatzes beide Briefe ungekürzt wiedergegeben.

Der ganzen Diskussion um die Vorgänge anläßlich der Veröffentlichung der Marxschen Schrift mit der Engelsschen Einleitung, so engagiert sie auch geführt wurde, fehlte immer die wichtigste quellenmäßige Grundlage, nämlich jener Brief, in dem Engels auf das Ansinnen des Parteivorstandes, angesichts der drohenden Umsturzvorlage einige Stellen aus seiner Einleitung zu streichen bzw. zu mildern, geantwortet hat. Schon Gustav Mayer hat bei den Vorarbeiten zu seiner Engels-Biographie vergeblich nach diesem Schreiben geforscht, ${ }^{1}$ und seitdem galt es als nicht auffindbar. ${ }^{2}$ Aus diesem Grunde mußte der Inhalt des Engelsschen Briefes bisher aus dem Schreiben Bebels an Engels vom 11. März $1895^{3}$ und dem Brief Fischers an Engels vom 14. des Monats erschlossen werden.

Fünf Quellen standen in der Vergangenheit zur Klärung der Vorgänge zur Verfügung:

1. Das Manuskript der Einleitung.

2. Die Setzereifahnen der Einzelausgabe von 1895.

3. Der Brief von Richard Fischer an Engels vom 6. März 1895, in dem er Engels im Auftrage des Parteivorstandes bittet, die von diesem vorgeschlagenen Korrekturen zu genehmigen.

4. Der Brief Bebels an Engels vom 11. März, in dem er sich auf dessen Schreiben an Fischer bezieht und Engels, der offensichtlich harte Kritik an der Taktik der Parteiführung geäußert hat, beschwichtigt. 5. Der Brief Fischers an Engels vom 14. März, in dem Fischer einmal Engels dafür dankt, daß er sich Korrekturvorschlägen des Vorstandes angeschlossen hat, zum anderen sich mit kritischen Bemerkungen des "Generals" auseinandersetzt. Auffällig ist, da B Fischer die Antwort solange herausgezögert hat, und man wird daher annehmen können, $\mathrm{da} B$ er sich bei den Mitgliedern des Vorstandes Rat geholt hat, vor allem bei Ignaz Auer, dem er sehr nahestand.

Aus der vorangegangenen Aufzählung ergibt sich, daß frühestens am 7., spätestens am 10. März Engels einen ausführlichen Brief an Fischer geschrieben haben muß.

1 Vgl. Gustav Mayer, Friedrich Engels, Bd. II, Den Haag 1934, S. 567.

2 Siehe hierzu Karl Marx und Friedrich Engels, Werke, Bd. 22, Berlin (O) 1963, S. 644, Anm. 433.

3 August Bebels Briefwechsel mit Friedrich Engels, hrsg. von Werner Blumenberg, 's Gravenhage 1965, S. 795-797. 
In der Tat datiert dieses Schreiben vom 8. März. Zwar scheint das Original verschwunden zu sein, aber im IISG befindet sich eine bislang unbeachtet gebliebene maschinenschriftliche Kopie dieses Briefes. Bis vor zwei Jahren war sie unter der Nr. MF I 124 im MarxEngels-NachlaB registriert, seitdem ist sie (nach der allgemeinen Neuordnung dieses Nachlasses) unter der Nr. K 513 dort zu finden. Werner Blumenberg, dem verstorbenen Leiter der Deutschlandabteilung des Instituts, gebührt das Verdienst, dieses wichtige Schreiben Engels' wiederentdeckt zu haben. Im Vorwort zu dem von ihm herausgegebenen Briefwechsel zwischen Engels und Bebel hat er einige Passagen daraus abgedruckt. ${ }^{1}$ Zweifellos hat er die Absicht gehabt, diesen Brief vollständig zu veröffentlichen, aber sein plötzlicher Tod hat dieses Vorhaben - wie auch andere umfassendere Pläne - zunichte gemacht.

Die Kopie umfaßt drei mit Schreibmaschine beschriebene Seiten. Papier und Schrifttypen lassen darauf schließen, daß sie aus den zwanziger Jahren, wenn nicht aus den ersten zwei Jahrzehnten des Jahrhunderts stammt. Auf der ersten Seite links unter dem oberen Rand findet sich folgende handschriftliche Notiz: „Ubergeben von Rich. Fischer an A. S. [Alexander Stein?] im Jahre 1924/25."

Angesichts der Tatsache, daß bezüglich der hier zur Diskussion stehenden Vorgänge noch heute von Fälschung die Rede ist, ${ }^{2}$ und im Hinblick auf die nicht immer korrekten Praktiken der an der Auseinandersetzung Beteiligten, lag es nahe, ein solches Dokument gerade weil das Original fehlt - mit stärkstem Mißtrauen zu betrachten und einer intensiven wissenschaftlichen Kritik zu unterziehen. Dabei hat sich ergeben:

1. Es ist ausgeschlossen, daß es sich bei dem vorliegenden Dokument, das sich überaus kritisch mit der Taktik der Parteiführung angesichts der Umsturzvorlage befaßt, um eine Fälschung von Apologeten des Vorstandes handelt. Im übrigen erlaubt der Inhalt des Briefes auch kaum die Bernsteinsche Interpretation der Engelsschen Einleitung. 2. Inhalt und Diktion des Schreibens weisen zweifelsfrei auf Friedrich Engels als den Verfasser.

Doch soll im folgenden der Engelssche Brief selbst für sich sprechen. Mit ihm werden aus den oben angeführten Gründen und um den Zusammenhang, in dem er steht, deutlich zu machen, die Briefe

1 Ebenda, S. XL, Anm. 57. Im übrigen kann ich - wie im folgenden ersichtlich ist - nicht der Interpretation Blumenbergs folgen, der eine grundsätzliche Übereinstimmung zwischen Engels auf der einen und Bebel sowie dem Parteivorstand auf der anderen Seite als gegeben annimmt.

2 Nach den relativ sachlichen Anmerkungen in Marx-Engels, Werke, a.a.O., Bd. 22, S. 644f. jetzt wieder in Bd. 2 der Geschichte der deutschen Arbeiterbewegung, Berlin (O) 1966, S. 59. 
Fischers abgedruckt. Dabei ist zu beachten, daß die Schreibweise modernisiert und die Interpunktion nach den heute gültigen Regeln behandelt wurde. Die häufig abgekürzte Konjunktion ,und" wird ständig ausgeschrieben, und Unterstreichungen der Verfasser werden durch Kursivdruck wiedergegeben. Einige Anstreichungen von fremder Hand in der Kopie des Engels-Briefes bleiben unberücksichtigt.

\section{RICHARD FISCHER AN FRIEDRICH ENGELS}

Berlin, [den] 6. März 1895

Lieber „General”!

Schon beim Durchlesen des Manuskriptes Deiner Einleitung hatte ich Bedenken gegen einzelne Passagen, und zwar waren es Bedenken mehr parteipolitischer als strafgesetzlicher Art. Ich wollte aber erst die Korrektur abwarten und habe jetzt mit August ${ }^{1}, \mathrm{Paul}^{2}$ und $\mathrm{Nazi}^{3}$ Rücksprache genommen. Wir haben uns über die hier beiliegende Korrektur geeinigt und möchten Dich bitten, Dich unserer Auffassung anzuschließen.

Wir gingen bei unserem Zensorenamte ${ }^{4}$ von dem Gedanken aus, daß die „beanstandeten" Stellen sehr leicht auf die Mühle unserer Gegner geleitet werden könnten. Und das müssen wir gerade heute besonders zu verhüten ${ }^{5}$ trachten. Wenn nicht alles trügt, scheint die Umsturzvorlage $\mathrm{zu}$ "versumpfen" - und insofern bist Du gegen die Rintelen und Spahn ${ }^{6}$ sehr grausam -.

Nun wirst $\mathrm{Du}$ aber selber zugeben, daß es einem böswilligen Gegner nicht schwer werden dürfte, als Quintessenz Deiner Ausführungen zu präsentieren 1) das Geständnis, daß wir bloß deshalb heute noch keine Revolution machen, weil wir noch nicht stark genug sind, weil das Heer noch nicht genügend infiziert ist - quod erat demonstrandum fïr die Umsturzvorlage; und zweitens, da $B$ wir im Falle eines Krieges

1 Bebel.

Singer.

Ignaz Auer.

"Die Stelle „bei unserem Zensorenamte” wurde von Bernstein ausgelassen, ohne daB diese Auslassung als solche kenntlich gemacht wurde.

"Von Bernstein fälschlich als ,vermeiden” entziffert.

- Dr. Victor Rintelen, Reichstagsabgeordneter des Zentrums; Dr. Peter Spahn, Zentrumspolitiker, Mitglied des Reichstages, 1917-1918 preuB. Justizminister. Zwischen der ersten Beratung der Umsturzvorlage im Reichstag im Dezember 1894 und im Januar 1895 sowie der zweiten Beratung im Maj 1895 wurde in der Kommission unter maßgeblichem Einfluß des Zentrums die Regierungsvorlage zu einem Gesetzentwurf zum Schutze von Sitte und Religion umgemodelt. Spahn war einer der Wortführer des Zentrums in der Kommission. 
oder sonstiger schwerer Verwicklungen - analog der Kommune - die Fahne des Aufruhrs angesichts des Landesfeindes etc. erheben würden.

Solches "Material” wäre heute gerade "gefundenes Fressen”, und alle Erklärungen unsererseits würden nur als Entschuldigungs- oder Ableugnungsversuche aufgefaßt werden. ${ }^{1}$

Ich glaube, $\mathrm{Du}$ wirst unsere Bedenken als berechtigt anerkennen müssen. Inzwischen mit herzl[ichen] Grüßen allseits

Dein R. Fischer

Bald hätte ich die Titelfrage vergessen: ich möchte mich entscheiden für den ersten Deiner drei Vorschläge

Die Klassenkämpte in Frankreich 1848-50 usw.

Du hast wohl auch die Güte und besorgst das mit den Korrekturen nach Hamburg.

Dann wäre noch eine Frage: $\mathrm{Ob}$ die zahlreichen französischen Zitate nicht gleich im Texte (in Parenthese) verdeutscht werden sollen, da schließlich doch die Arbeiter zu 99\% Käufer und Leser sein werden? Was meinst Du dazu?

\section{FRIEDRICH ENGELS AN RICHARD FISCHER}

8. März [18]95

41, Regent's Park Road N.W.

\section{Lieber Fischer,}

Ich habe Euren schweren Bedenken nach Möglichkeit Rechnung getragen, obwohl ich beim besten Willen nicht einsehen kann, worin die Bedenklichkeit bei etwa der Hälfte besteht. Ich kann doch nicht annehmen, daß Ihr Euch mit Leib und Seele der absoluten Gesetzlichkeit, der Gesetzlichkeit unter allen Umständen, der Gesetzlichkeit auch gegenüber den von ihren Urhebern gebrochenen Gesetzen, kurz der Politik des Hinhaltens der linken Backe dem, der auf die rechte gehauen hat, zu verschreiben beabsichtigt. Im „Vorwärts” wird die Revolution allerdings manchmal mit ebensoviel Kraftaufwand verleugnet, wie früher - vielleicht auch nächstens wieder gepredigt. Aber das kann ich doch nicht für maßgebend halten.

1 Hier endet der Bernsteinsche Abdruck. 
Ich bin der Ansicht, daß Ihr nichts dadurch gewinnt, wenn Ihr den absoluten Verzicht aufs Dreinschlagen predigt. Glauben tuts kein Mensch, und keine Partei irgendeines Landes geht so weit, auf das Recht zu verzichten, der Ungesetzlichkeit mit den Waffen in der Hand zu widerstehn.

Auch muß ich darauf Rücksicht nehmen, daß auch Ausländer Franzosen, Engländer, Schweizer, Österreicher, Italiener etc. - meine Sachen lesen, und vor denen kann ich mich platterdings nicht so weit kompromittieren.

Ich habe also Eure Änderungen akzeptiert mit folgenden Ausnahmen: 1.) Fahne 9, bei den Massen, heißt es jetzt: sie müssen begriffen haben, für was sie eintreten sollen. ${ }^{1}-2$.) folgender Absatz: der ganze Satz vom Losschlagen gestrichen. ${ }^{2}$ Euer Vorschlag enthielt eine tatsächliche Unrichtigkeit. Das Schlagwort vom Losschlagen ${ }^{3}$ gebrauchen Franzosen, Italiener etc. alle Tage, nur ists weniger Ernst. 3.) Fahne 10: Der sozialdem[okratische] Umsturz, der augenblicklich davon lebt, wollt Ihr das augenblicklich fort, also eine augenblickliche in eine dauernde, eine relative in eine absolut geltende Taktik verwandeln. Das tue ich nicht, kann ich nicht tun, ohne mich unsterblich zu blamieren. Ich vermeide also die Stellung des Gegensatzes und sage: der $\mathrm{s}[\mathrm{o}] \mathrm{z}$ [ialdemokratische] Umsturz, dem es gerade jetzt sehr gut bekommt, daß er die Gesetze hält. ${ }^{4}$

Warum Ihr den Hinweis auf Bismarcks Vorgang 1866 beim Verfassungsbruch gefährlich findet, ist mir absolut unerfindlich. Das ist doch ein argumentum ad hominem wie kein zweites. Jedoch, ich tue Euch den Gefallen. ${ }^{5}$

So, weiter kann ich aber absolut nicht gehen. Ich habe mein Möglichstes getan, Euch Ungelegenheiten in der Debatte zu ersparen. Aber Ihr tätet besser, den Standpunkt zu wahren, daß die Verpflichtung zur Gesetzlichkeit eine juristische, keine moralische ist, wie Euch das

1 Im ursprüngl. Manuskript heißt es: „Wo es sich um eine vollständige Umgestaltung der gesellschaftlichen Organisation handelt, da müssen die Massen selbst mit dabei sein, selbst schon begriffen haben, worum es sich handelt, für was sie mit Leib und Leben eintreten". Der Vorstand wollte wohl den letzten Teil des Satzes streichen, Engels ersetzte ihn durch „für was sie eintreten sollen”.

2 Es handelt sich um die Stelle des Manuskripts: „... überall ist das unvorbereitete Losschlagen in den Hintergrund getreten."

s Es läßt sich rekonstruieren, daß der Vorstand Engels ungefähr folgende Version vorgeschlagen hat: , . . . iberall ist das Schlagwort vom unvorbereiteten Losschlagen in den Hintergrund getreten."

4 Vgl. hierzu den Text und die 2. Fassung in Marx-Engels, Werke, a.a.O., Bd. 22, S. 525.

Vgl. ebenda, S. 526. 
der Boguslawski ${ }^{1}$ (der ein langes $s$ hat) ${ }^{2}$ ja so schön vorgemacht hat; und daß sie vollends aufhört, wenn die Machthaber die Gesetze brechen. Ihr habt aber - oder wenigstens dieser und jener unter Euch die Schwäche gehabt, dem Anspruch der Gegner nicht gehörig entgegenzutreten: die Verpflichtung zur Gesetzlichkeit auch als eine moralische anzuerkennen, als eine unter allen Umständen bindende, statt zu sagen: Ihr habt die Macht, ihr macht die Gesetze, übertreten wir sie, so könnt ihr uns nach diesen Gesetzen behandeln, wir müssen das ertragen und damit ists aus, wir haben weiter keine Pflicht, ihr weiter kein Recht. So habens die Katholiken gemacht unter den Maigesetzen, so die Altlutheraner in Meißen, so jener mennonitische Soldat, der in allen Zeitungen figuriert, ${ }^{3}$ und den Standpunkt dürft Ihr nicht verleugnen. Die Umsturzvorlage geht sowieso in die Brüche, so was ist gar nicht zu formulieren und noch weniger durchzuführen, und haben die Leute die Macht, so knebeln und zwiebeln sie Euch doch in irgendeiner Weise.

Wenn Ihr aber den Leuten in der Regierung klar machen wollt, da $B$ wir nur deshalb warten wollen, weil wir noch nicht stark genug sind, uns selbst zu helfen, und weil die Armee noch nicht gründlich verseucht ist - ja, dann liebe Leute, warum renommiert Ihr dann alle Tage in den Blättern mit den Riesenfortschritten und Erfolgen der Partei? Die Leute wissen ja so gut wie wir, daß wir mit Macht dem Sieg entgegenrücken, da $B$ wir in ein paar Jahren unwiderstehlich werden, und deswegen wollen sie uns schon jetzt an den Kragen, leider wissen sie nicht wie. Daran können unsre Reden nichts ändern, das wissen sie alles so gut wie wir, und ebenso wissen sie, daß, haben wir die Macht, wir sie gebrauchen werden, wie's uns dient und nicht ihnen.

Also wenns zur Generaldebatte im Plenum kommt, denkt a bissel daran, daß Ihr das Recht des Widerstands ebensogut wahrt, wie es Boguslawski uns gewahrt hat, daß Ihr auch alte Revolutionäre,

I Albert von Boguslawski (1834-1905), Offizier und Militärschriftsteller, unterdrückte 1889 als Kommandeur der 21. Infanteriebrigade in Schlesien im Zusammenhang mit dem Bergarbeiterstreik ausgebrochene Unruhen mit Waffengewalt. 1891 wurde er im Range eines Generalleutnants verabschiedet. Engels bezieht sich hier mit größter Wahrscheinlichkeit auf die Anfang 1895 erschienene Schrift Boguslawskis ,Vollkampf nicht Scheinkampf. Ein Wort zur politischen Lage im Innern", die gegen die Sozialdemokratie gerichtet war und den Staatsstreich von oben propagierte.

2 Auf den in Fraktur gesetzten Druckfahnen (Marx-Engels-Nachlaß, H 153) findet sich einmal (Fahne 10) der Name Boguslawski mit „Schluß-s”, die entsprechende Stelle ist von Engels korrigiert.

3 Engels bezieht sich hier auf den Fall des Grenadiers Thröner, welcher den Dienst mit der Waffe verweigert hatte. Thröner gehörte der radikalen Mennonitengemeinschaft der „Fröhlichianer” an. Zum „Fall Thröner” vgl. Theologischer Jahresbericht, Bd. 15, 1895, Braunschweig 1896, S. 337. 
Franzosen, Italiener, Spanier, Ungarn, Engländer unter Euren Zuhörern habt und daß - wer weiß wie bald - die Zeit wieder kommen kann, wo Ernst gemacht wird mit der Streichung des "gesetzlich", die anno Tobak in Wyden vollzogen wurde. ${ }^{1}$ Seht doch die Österreicher an, die so direkt wie möglich mit der Gewalt drohen, wenns Wahlrecht nicht bald kommt!2 Denkt an Eure eigenen Ungesetzlichkeiten unter dem Soz[ialisten]gesetz, das man Euch wieder anhängen möchte! Gesetzlichkeit solange und soweit sie uns paßt, aber keine Gesetzlichkeit um jeden Preis, selbst nicht in der Phrase!

Dein Fr. E.

Verdeutschung der Zitate - die meisten sinds schon im Text - jetzt zu spät, schon im Bogen umgebrochen.

\section{Die Korrektur geht nach Hamburg von hier.}

\section{RICHARD FISCHER AN FRIEDRICH ENGELS}

Berlin, [den] 14. März 1895

\section{Lieber „General”!}

Besten Dank für Deine Bereitwilligkeit für unsere Korrekturvorschläge. Du gehst aber bei Deinen Bemerkungen von ganz falschen Voraussetzungen aus. Es fällt keinem von uns ein, „uns mit Leib und Seele der Gesetzlichkeit zu verschreiben"3 etc., sowenig als wir daran denken, „den absoluten Verzicht aufs Dreinschlagen zu predigen”.

Es würde uns das - da hast Du auch ganz recht - niemand glauben, am allerwenigsten jetzt gerade. Wenn nun das also auch ein albernes Spiel wäre, so wäre es doch auch wieder ebenso töricht, wollten wir gerade jetzt unsere Stärke darin suchen, dem Gegner, der uns mit der Umsturzvorlage an der Gurgel kniet, immerfort die Drohung zuzuschreien: Warte nur, wenn ich erst mal mich wieder rühren kann, dann

1 Auf dem Wydener Kongreß 1880 wurde auf Antrag Schlüters aus Absatz II des Gothaer Programms - ,Von diesen Grundsätzen ausgehend, erstrebt die Sozialistische Arbeiterpartei Deutschlands mit allen gesetzlichen Mitteln den freien Staat und die sozjalistische Gesellschaft, die Zerbrechung des ehernen Lohngesetzes durch Abschaffung des Systems der Lohnarbeit, die Aufhebung der Ausbeutung in jeder Gestalt, die Beseitigung aller sozialen und politischen Ungleichheit" - das Wort „gesetzlich" gestrichen.

2 Betr. die seit 1893 mit aller Schärfe geführten Wahlrechtskämpfe in Österreich. Bebel wies im Brief vom 11. März jeden Vergleich zwischen der Situation in Österreich und der in Deutschland als unzulässig zurück.

${ }^{3}$ Alles weitere bis zum SchluB des Absatzes wurde von Bernstein nicht veröffentlicht. Die Auslassung ist von ihm kenntlich gemacht worden. 
schneide $i c h$ Dir die Gurgel glattweg ab. $-^{1}$ Nein, wir halten es da mit jenem vernünftigen Bauernmädel, die ihrem täppisch-zagenden Burschen einfach erklärte: Von so was redet man nicht, so was tut man!²

Du tust uns wohl auch darin Unrecht, daß Du annimmst, wir hätten uns von den Gegnern dazu drängen lassen, die Verpflichtung zur Gesetzlichkeit auch als eine moralische anzuerkennen. Das ist von keiner Seite geschehen; im Gegenteil, vom Alten ${ }^{3}$ sowohl wie von August ${ }^{4}$ ist gerade in jüngster Zeit mit aller Schärfe wiederholt betont worden, daß Verfassungs- und Gesetzesbruch von oben nach unten alle Verpflichtungen von unten nach oben aufhebe.

Wir sind heute gesetzlich, weil es in unserem Vorteil liegt und nebenbei - die anderen auch noch stark genug sind, uns dazu zwingen zu können; und wir berufen uns auf diese Gesetzlichkeit sogar jetzt, weil dies gerade heute unseren Gegnern besonders unangenehm ist und ihnen ihr Konzept verdirbt.

Voilà tout $!^{5}$

Und Du wirst auch sehen, daß wir den Franzosen, Italienern usw. keine Gelegenheit geben werden, nach der Richtung hin über uns die Nase zu rümpfen, sowenig als wir vergessen oder verleugnen, daß wir in Wyden das "gesetzlich" aus dem Programm gestrichen und in Erfurt es nicht mehr aufgenommen haben.

$\mathrm{Im}$ übrigen weißt $\mathrm{Du}$ das alles auch, sonst hättest $\mathrm{Du}$ ja Deine $\mathrm{Zu}$ stimmung $\mathrm{zu}$ den Korrekturen nicht gegeben. ${ }^{6}$

Mit herzlichen Grüßen

Dein R. Fischer

Aus dem Engelsschen Schreiben geht hervor, daß er mit Rücksicht auf die bevorstehende Debatte über die Umsturzvorlage die vom Parteivorstand vorgeschlagenen Änderungen im Text seiner Einleitung mit Ausnahme der im Brief aufgeführten relativ belanglosen Stellen akzeptiert hat. Das ist eine nicht zu leugnende Tatsache. Allerdings kann in keiner Weise von einer Zustimmung seinerseits zur Taktik

1 Das folgende bis zum Schluß des Absatzes wurde von B. ausgelassen ohne irgendeinen Hinweis darauf.

2 Dieser Vergleich stammt augenscheinlich von dem mit Fischer eng befreundeten Ignaz Auer. Im Revisionismusstreit von Auer auf Bernsteins Initiative angewandt, wurde der Satz „So was sagt man nicht, so was tut man” zum Programm der Praktiker in der Partei.

3 Wilhelm Liebknecht.

4 Bebel.

- Der ganze vorhergehende Absatz wurde von B. nicht abgedruckt.

- Hier endet der Bernsteinsche Abdruck. 
der Partei, die seit Ende 1894 mit ihrem legalen Verhalten in der Offentlichkeit geradezu paradierte, die Rede sein. Es erscheint gerade im Hinblick auf den vorliegenden Brief als notwendig, die von Engels propagierte Taktik der Gesetzlichkeit, bei der die Partei „pralle Muskeln und rote Backen"1 bekommen sollte, und das Taktieren der Parteiführung genauer in den Blick zu nehmen.

Friedrich Engels war schon in den achtziger Jahren davon überzeugt, daß die an den Wahlergebnissen abzulesende Entwicklung der Partei in einer Weise fortschreiten werde, „daß die Gleichung ihrer wachsenden Geschwindigkeit und damit der Zeitpunkt ihres schließlichen Sieges sich schon jetzt mathematisch berechnen läßt."2 Die Engelsschen Erwartungen waren - und dadurch unterschied er sich von den Repräsentanten der deutschen Partei - Ausdruck einer revolutionären Grundkonzeption. Der Gegenstand, um den sich seine Spekulationen in erster Linie bewegten, waren die „Kernregimenter” der preußischen Armee. Er hielt eine Revolution angesichts der waffentechnischen Entwicklung nur dann für aussichtsreich, wenn die Mehrheit der Armee sozialistisch sei. ${ }^{3}$ Die Überschätzung des Tempos der Entwicklung und die Fehlkalkulation hinsichtlich der Gewinnung der pommerschen und mecklenburgischen Landbevölkerung brachten ihn Anfang der neunziger Jahre dahin, mit Sicherheit anzunehmen, daß um die Jahrhundertwende die Armee in Deutschland den herrschenden Klassen „entschlüpft” sei. ${ }^{4}$ Durch zwei Momente sah Engels die unaufhaltsame Entwicklung bedroht: durch ein vorzeitiges Losschlagen der Partei und durch einen europäischen Krieg. Schon lange vor der Abfassung der Einleitung von 1895 hat er vor einem Aderla $B$ wie dem von 1871 in Paris gewarnt. Der Gedanke, daß der Versuch, durch „Überrumpelung" die soziale und politische Umgestaltung zu erreichen, die Bewegung aufs schwerste schädigen würde, hat ihn seit dem Ende der

1 Marx-Engels, Werke, a.a.O., Bd. 22, S. 525.

${ }^{2}$ Engels an Kautsky, 8.11.1884, Fr. Engels' Briefwechsel mit Karl Kautsky, a.a.O., S. 154 .

${ }^{3}$ Schon im Anschlu $B$ an die Reichstagswahlen von 1877 hat Engels in einem Brief an Bignami, den Redakteur der Mailänder sozialistischen Zeitung La Plebe, seine Auffassung über die durch das Wahlergebnis beweisbare sozialistische Unterwanderung speziell der Heeresreserven dargelegt (Marx-Engels, Werke, a.a.O., Bd. 19, S. 89f.).

4 Fr. Engels, Der Sozialismus in Deutschland, Marx-Engels, Werke, a.a.O., Bd. 22, S. 251. Zusammenstellung aller Belege in meiner in der Schriftenreihe des Forschungsinstituts der Friedrich-Ebert-Stiftung erschienenen Arbeit "Sozialismus und deutsche Sozialdemokratie. Zur Ideologie der Partei vor dem 1. Weltkrieg". 
siebziger Jahre nicht mehr losgelassen. ${ }^{1}$ Unter dem Eindruck der erfolgreichen Benutzung des allgemeinen Stimmrechts durch die deutsche Sozialdemokratie glaubte er, in diesem das eigentliche revolutionäre Mittel im Gegensatz zu den veralteten Barrikadenkämpfen etwa der Kommune sehen zu müssen.

Das, was die Mehrheit in der Sozialdemokratie, insbesondere später die Revisionisten, von Engels übernahm, war die Betonung des gesetzlichen und friedfertigen Charakters der Partei. Schon 1890 sah sich Engels veranlaßt, bei Liebknecht dagegen zu protestieren, daß seine Mahnung, durch voreilige Aktionen nicht das „eigene Spiel” zu verderben, in ein generelles Bekenntnis zur Gewaltlosigkeit umgemünzt wurde. ${ }^{2}$ Gerade aus dem Brief vom 8. März 1895 wird der Unterschied zwischen seiner Auffassung und der des Parteivorstandes deutlich. Wie recht Engels hatte, als er die Haltung der Partei als die "Politik des Hinhaltens der linken Backe dem, der auf die rechte gehauen hat", kennzeichnete, sollte sich ein Jahr später herausstellen, als in der Hochburg der Sozialdemokratie, in Sachsen, ein Dreiklassenwahlrecht eingeführt wurde und die Partei in gemäßigtem Protest verharrte. Engels, der der Partei die Osterreicher als Vorbild hinstellte, hat gespürt, daß seine von der revolutionären Erwartung bestimmte Anschauung in der Sozialdemokratie im Sinne einer Gesetzlichkeit um jeden Preis interpretiert wurde. Bebels Worte im Reichstag: „Bis auf den heutigen Tag können Sie der Sozialdemokratie gewaltsame revolutionäre Bestrebungen, den geringsten Versuch, auf gewaltsamem revolutionärem Wege ihre Ziele verwirklichen zu wollen, nicht nachweisen", ${ }^{3}$ können nicht nur als taktische Äußerungen angesichts der Umsturzvorlage interpretiert werden, sie sind vielmehr Ausdruck des wahren Charakters der Partei. ${ }^{4}$ Bebel und Fischer haben versucht, Engels zu beschwichtigen, und gerade Bebels Brief ist ungemein aufschlußreich: ,... wir haben gar keinen $Z$ weifel darüber gelassen, daß der natürliche Lauf der Entwicklung uns die Macht in die Hände liefere, über das Wie haben wir uns nicht ausgesprochen, wir haben nur bestritten, daB wir Neigung hätten, mit den neuen Repetiergewehren Bekanntschaft zu machen."5 Auf das „Wie” aber kommt es an, und wie zum

1 Vgl. den Brief an Bignami, den Aufsatz „Der Sozialismus in Deutschland”, den Beitrag „Was nun?" in der Festnummer des „Sozialdemokrat" anläßlich der Wahl (Nr. 10, 8.3.1890) sowie den Brief an Liebknecht vom 9.3.1890 (W. Liebknecht, Briefwechsel mit Karl Marx und Friedrich Engels, hrsg. und bearb. von Georg Eckert, Den Haag 1963, S. 366).

Engels an Liebknecht, 9.3.1890, ebenda, S. 367.

sten. Ber., IX. Leg. III. Sess., 1894-95, Bd. 3, S. 2172 f.

4 Vgl. z.B. die Ausführungen Grillenbergers vom Februar 1891 im Reichstag (Sten. Ber. VIII. Leg., I. Sess. 1890-91, Bd. 3, S. 1776, 1805).

s Bebel an Engels, 11.3.1895, a.a.O., S. 795f. 
Beispiel Auer, neben Bebel der Hauptredner in den parlamentarischen Debatten um die Umsturzvorlage, der im Reichstag Engelssche Gedanken bezüglich der gesetzlichen Taktik vortrug, sich den Übergang zum Sozialismus vorstellte, das hatte mit Engels' Konzeption nur wenig gemein. Zwischen dieser und den Vorstellungen des Parteivorstandes bestand ein grundlegender Unterschied, der in der Perspektive lag. Die Versuche schließlich, die Engelssche Einleitung zur Begründung des Revisionismus heranzuziehen, beruhten auf der falschen Ansicht, daß Engels' Absage an die revolutionäre Taktik hergebrachter Art eine grundsätzliche Abkehr von der Idee der proletarischen Revolution markiere. Engels, der davon ausging, da $B$ bei einem vorläufig streng gesetzlichen Verhalten der Partei die Regierung und die durch sie repräsentierte Gesellschaft tatenlos der unwiderstehlichen Aufwärtsentwicklung ihrer Todfeinde zusehen müßten, bis eines Tages die Quantität der sozialistischen Mehrheit zwangsläufig zum revolutionären Umschlag führen würde, der, weil auch das Gros der bewaffneten Macht auf seiten der revolutionären Bewegung stehen würde, mit dem vollständigen Sieg enden müßte, verkannte lange den scheinrevolutionären Charakter der deutschen Partei. Erst kurz vor seinem Tod scheint ihm aufgegangen zu sein, daß für die Partei das Bekenntnis zur Gesetzlichkeit mehr war als eine bloße Phrase.

Für Engels und die Parteiführung war gleichermaßen der Endsieg exakt berechenbar, und daher durfte die zwangsläufige Entwicklung nicht gestört werden. Die Engelssche Taktik des Stillhaltens und Abwartens aber blieb Ausdruck eines realistischen Kalküls im Hinblick auf die Möglichkeit der sozialen und politischen Revolution. Demgegenüber sah man in der deutschen Partei einmal die Fortentwicklung zur parlamentarischen Mehrheit, wobei die proletarische Revolution aus diesem Entwicklungsglauben eliminiert wurde, als Ziel an, zum anderen erwartete man (Bebel!) in fatalistischer Weise einen Zusammenbruch der bestehenden Ordnung. 